\title{
Ancient Chinese constellations
}

\author{
Junjun Xu \\ Beijing University of Aeronautics and Astronautics \\ Room 424, Apartment 20, No. 37 Xueyuan Street, Beijing, China \\ email: xjjhhyy@sina.com
}

\begin{abstract}
China, a country with a long history and a specific culture, has also a long and specific astronomy. Ancient Chinese astronomers observed the stars, named and distributed them into constellations in a very specific way, which is quite different from the current one. Around the Zodiac, stars are divided into four big regions corresponding with the four orientations, and each is related to a totem, either the Azure Dragon, the Vermilion Bird, the White Tiger or the Murky Warrior. We present a general pattern of the ancient Chinese constellations, including the four totems, their stars and their names.
\end{abstract}

Keywords. China, constellations, mansions

\section{Introduction}

Three enclosures, four symbols and twenty-eight mansions characterise the ancient Chinese constellations. This division of the starry sky began to appear in China before the Zhou and Qin dynasties. The three enclosures refer to three areas around the North celestial pole: the Purple Forbidden enclosure, the Supreme Palace enclosure and the Heavenly Market enclosure. The four symbols are distributed near the ecliptic Zodiac and the lunar orbit and are represented by four totems: the Azure Dragon of the East, the Vermillion Bird of the South, the White Tiger of the West and the Black Tortoise of the North. Every symbol was divided into seven sections which were know as mansions. There are twenty eight mansions in this system and the Moon goes through approximately a mansion every day, reflecting the lunar month. The twenty eight mansions $†$ were named Horn, Dipper, Legs, Well, Neck, Ox, Bond, Ghosts, Root, Girl, Stomach, Willow, Room, Emptiness, Hairy head, Star, Heart, Rooftop, Net, Extended net, Tail, Encampment, Turtle beak, Wings, Winnowing-basket, Wall, Three stars, Chariot, as show in Fig. 1.

\section{Tables of star names}

The following tables are a comparison list of star names both in traditional Chinese characters and in English. They are classified in seven tables according to the three enclosures and the four animal images which they belong toł.

\section{Implications}

Ancient Chinese astronomy has taken an important rôle in both the Chinese culture and the world astronomical culture. Chinese ancestors divided the constellations and denominated the stars in a quite different way as the Greeks did. It will be an interesting

$\dagger$ see also http://baike.baidu.com/view/17723.htm?fr=ala0_1_1

$\ddagger$ http://www.lcsd.gov.hk/CE/Museum/Space/Research/StarName/c_research_ chinengstarzone_b.htm 


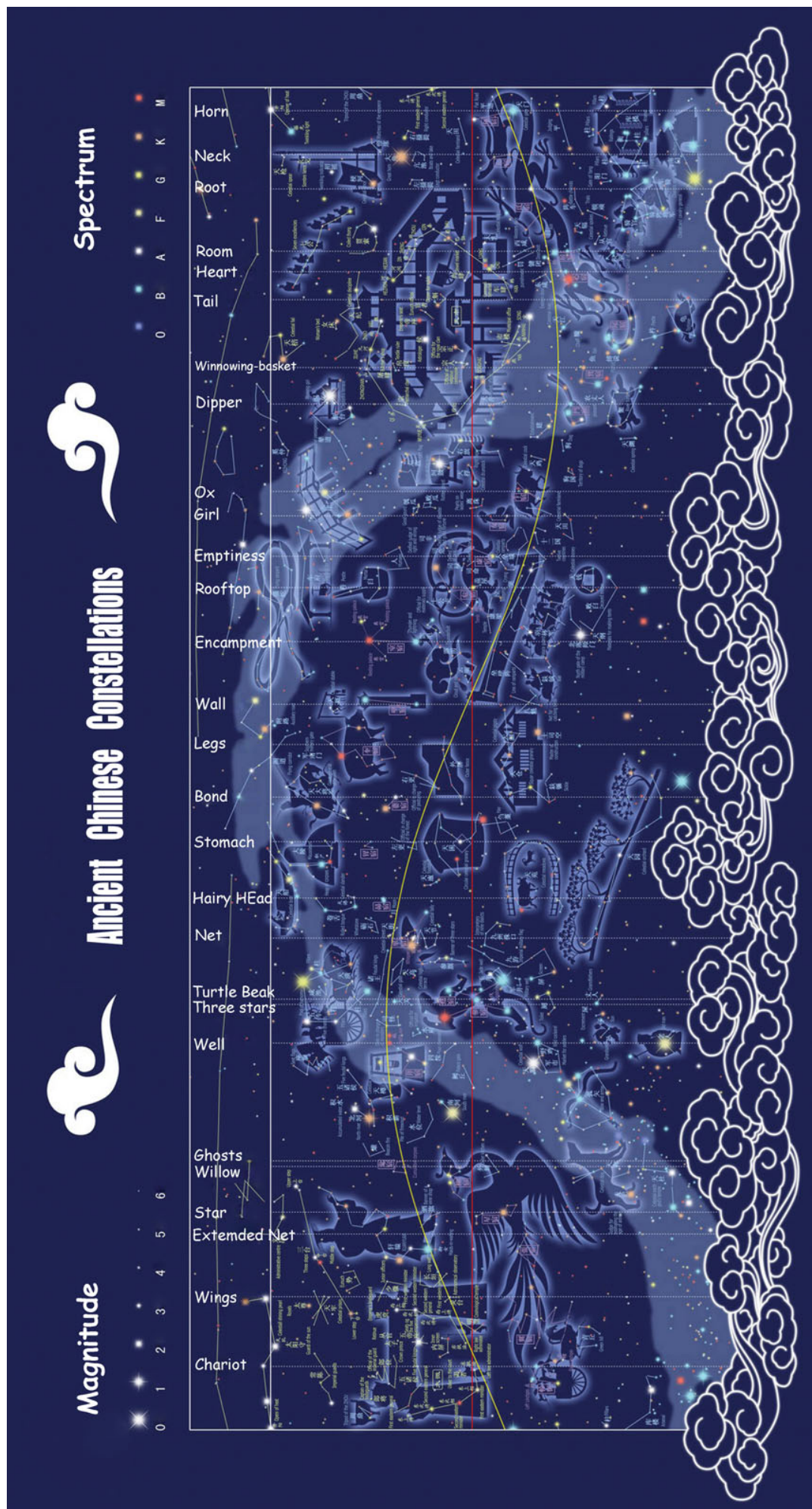

Figure 1. Ancient Chinese constellations. Artwork by Zuojing Guanxing. 
Table 1. The First Enclosure: Purple Forbidden Enclosure

\begin{tabular}{|c|c|c|c|c|c|}
\hline \multicolumn{6}{|c|}{ 紫微垣 Purple Forbidden Enclosure } \\
\hline 北極 & Northern pole & 紫微左垣 Left wall & & 紫微右垣 & Right Wall \\
\hline 太子 & Crown prince & 左樞 Left pivot & 右樞 & Right pivot & 三公 $\begin{array}{l}\text { Three } \\
\text { excellencies }\end{array}$ \\
\hline 帝 & Emperor & 少宰 Second premier & 少尉 & $\begin{array}{l}\text { Second chief } \\
\text { judge }\end{array}$ & Prime minister \\
\hline 庶子 & $\begin{array}{l}\text { Son of Concu- } \\
\text { bine }\end{array}$ & 上咟 First minister & 上博 & First minister & 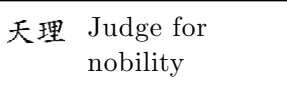 \\
\hline 後宫 & $\begin{array}{l}\text { Imperial } \\
\text { Concubine }\end{array}$ & 少弼 Second minister & 少博 & Second minister & 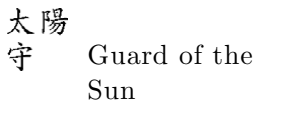 \\
\hline 天樞 & Celestial pivot & $\begin{array}{l}\text { 上衛 } \begin{array}{l}\text { First imperial } \\
\text { guard }\end{array}\end{array}$ & | 上衛 & $\begin{array}{l}\text { First imperial } \\
\text { guard }\end{array}$ & 太尊 Royals \\
\hline 四輔 & Four Advisors & $\begin{array}{l}\text { 少衛 } \begin{array}{l}\text { Second Imperial } \\
\text { guard }\end{array}\end{array}$ & | 少衛 & $\begin{array}{l}\text { Second imperial } \\
\text { guard }\end{array}$ & 天牢 Celestial prison \\
\hline 勾陳 & Curved Array & $\begin{array}{l}\text { 少丞 } \begin{array}{l}\text { Second prime } \\
\text { minister }\end{array}\end{array}$ & 上丞 & $\begin{array}{l}\text { First prime } \\
\text { minister }\end{array}$ & 勢 Eunuch \\
\hline $\begin{array}{l}\text { 天皇 } \\
\text { 大帝 }\end{array}$ & $\begin{array}{l}\text { Great Emperor } \\
\text { of Heaven }\end{array}$ & 少宰 Second premier & 天乙 & $\begin{array}{l}\text { Celestial Great } \\
\text { One }\end{array}$ & $\begin{array}{l}\text { 文昌 Administrative } \\
\text { centre }\end{array}$ \\
\hline 天柱 & Celestial Pillar & |上唱 First minister & |太乙 & Frist Great one & 内階 Inner steps \\
\hline 御女 & $\begin{array}{l}\text { Maids-in- } \\
\text { waiting }\end{array}$ & 少弼 Second minister & 内廊 & Inner kitchen & $\begin{array}{ll}\text { 三師 } & \text { Three top } \\
& \text { instructors }\end{array}$ \\
\hline 女史 & Female protocol & $\mid \begin{array}{ll}\text { 上衛 } & \text { First imperial } \\
& \text { guard }\end{array}$ & 北斗 & Northern dipper & $\begin{array}{l}\text { 八穀 Eight kinds of } \\
\text { crops }\end{array}$ \\
\hline 柱史 & $\begin{array}{l}\text { Official of royal } \\
\text { archives }\end{array}$ & $\begin{array}{l}\text { 少衛 } \begin{array}{l}\text { Second Imperial } \\
\text { guard }\end{array}\end{array}$ & |天樞 & Celestial pivot & 傅舍 Guest house \\
\hline 尚書 & Royal secretary & $\begin{array}{l}\text { 少丞 } \begin{array}{l}\text { Second prime } \\
\text { minister }\end{array}\end{array}$ & 天璇 & $\begin{array}{l}\text { Celestial rotating } \\
\text { jade }\end{array}$ & $\begin{array}{c}\text { 天廚 } \begin{array}{l}\text { Celestial } \\
\text { kitchen }\end{array}\end{array}$ \\
\hline 天床 & Celestial bed & & 天機 & $\begin{array}{l}\text { Celestial shining } \\
\text { pearl }\end{array}$ & 天棓 Celestial flail \\
\hline 大理 & Chief judge & & |天權 & Celestial balance & \\
\hline 陰德 & Hidden virtue & & 玉衡 & $\begin{array}{l}\text { Jade } \\
\text { sighting-tube }\end{array}$ & \\
\hline 六甲 & Six Jia & & |開陽 & Opener of heat & \\
\hline $\begin{array}{l}\text { 五帝 } \\
\text { 内座 }\end{array}$ & $\begin{array}{l}\text { Interior seats of } \\
\text { five emperors }\end{array}$ & & 搖光 & $\begin{array}{l}\text { Twinkling } \\
\text { brilliance }\end{array}$ & \\
\hline 華蓋 & $\begin{array}{l}\text { Canopy of the } \\
\text { emperor }\end{array}$ & & & Assistant & \\
\hline 杠 & CanopySupport & & |玄戈 & Sombre lance & \\
\hline
\end{tabular}


Table 2. The Second Enclosure: Supreme Palace Enclosure

\begin{tabular}{|c|c|c|c|c|c|c|}
\hline \multicolumn{7}{|c|}{ 太微垣 Supreme Palace Enclosure } \\
\hline 五帝 & $\begin{array}{l}\text { Seats of the } \\
\text { five emperors }\end{array}$ & 太微 & 左垣 Left wall & \multicolumn{3}{|c|}{ 太微右垣 Right wall } \\
\hline 太子 & Crown prince & 上宰 & First premier & $\begin{array}{l}\text { 右執 } \\
\text { 法 }\end{array}$ & $\begin{array}{l}\text { Right law } \\
\text { administrator }\end{array}$ & 上台 Upper step \\
\hline 從官 & Retinue & $\begin{array}{l}\text { 東上 } \\
\text { 相 }\end{array}$ & $\begin{array}{l}\text { First eastern } \\
\text { minister }\end{array}$ & $\begin{array}{l}\text { 西上 } \\
\text { 將 }\end{array}$ & $\begin{array}{l}\text { First western } \\
\text { general }\end{array}$ & 中台 Middle step \\
\hline & $\begin{array}{l}\text { Officer of } \\
\text { honour }\end{array}$ & $\begin{array}{l}\text { 東次 } \\
\text { 相 }\end{array}$ & $\begin{array}{l}\text { Second eastern } \\
\text { minister }\end{array}$ & $\mid$\begin{tabular}{|l} 
西次 \\
將
\end{tabular} & $\begin{array}{l}\text { Second } \\
\text { western } \\
\text { general }\end{array}$ & 下台 ower step \\
\hline $\begin{array}{l}\text { 五諸 } \\
\text { 候 }\end{array}$ & $\begin{array}{l}\text { Five feudal } \\
\text { kings }\end{array}$ & $\begin{array}{l}\text { 東次 } \\
\text { 將 }\end{array}$ & $\begin{array}{l}\text { Second eastern } \\
\text { general }\end{array}$ & 西次 & $\begin{array}{l}\text { Second } \\
\text { western } \\
\text { minister }\end{array}$ & $\begin{array}{c}\text { 虎賁 } \begin{array}{l}\text { Emperor's } \\
\text { bodyguard }\end{array}\end{array}$ \\
\hline 九卿 & $\begin{array}{l}\text { Nine senior } \\
\text { officers }\end{array}$ & $\begin{array}{l}\text { 束上 } \\
\text { 將 }\end{array}$ & $\begin{array}{l}\text { First eastern } \\
\text { general }\end{array}$ & $\begin{array}{l}\text { 西上 } \\
\text { 相 }\end{array}$ & $\begin{array}{l}\text { First western } \\
\text { minister }\end{array}$ & 少微 Junior officers \\
\hline & $\begin{array}{l}\text { Three } \\
\text { excellencies }\end{array}$ & 上宰 & First premier & 郎將 & $\begin{array}{l}\text { Captain of the } \\
\text { bodyguards }\end{array}$ & 長垣 ong wall \\
\hline & & & & 郎位 & $\begin{array}{l}\text { Official of } \\
\text { imperial guard }\end{array}$ & 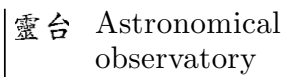 \\
\hline & & & & 常陳 & $\begin{array}{l}\text { Imperial } \\
\text { guards }\end{array}$ & \begin{tabular}{|l} 
明堂 $\begin{array}{l}\text { Cosmological } \\
\text { temple }\end{array}$
\end{tabular} \\
\hline & & & & 三台 & Three steps & $\begin{array}{l}\text { 謁者 Usher of the } \\
\text { court }\end{array}$ \\
\hline
\end{tabular}

work to carry out a comparison between these two systems. Ancient Chinese constellations were rooted in a strong belief of harmony among the heaven, the Earth and human beings. By studying the stories behind the the names of the stars, one learns ancient Chinese astronomy and also Chinese history and philosophy as well.

\section{References}

Chen, J. 2005, Ancient Chinese Constellations Decoding (Beijing: Qunyan Publisher)

Sun, X. \& Kistemaker, J. 1997, The Chinese sky during the Han: Constellating Stars and Society (Leiden: Brill) 
Table 3. The Third Enclosure: Heavenly Market Enclosure

\begin{tabular}{|c|c|c|c|c|}
\hline \multicolumn{5}{|c|}{ 天市垣 Heavenly Market Enclosure } \\
\hline 帝 & $\begin{array}{l}\text { Emperor's } \\
\text { seat }\end{array}$ & 天市左垣 Left wall & 天市右垣 & Right wall \\
\hline 候 & Astrologer & 魏 WEI & 河 $\mathrm{HEZHONG}$ & $\mid \begin{array}{ll}\text { 天 } & \\
\text { 紀 } & \text { Celestial } \\
& \text { Discipline }\end{array}$ \\
\hline $\begin{array}{l}\text { 宦 } \\
\text { 者 }\end{array}$ & Eunuch official & 趙 ZHAO & 河 & \begin{tabular}{|l} 
女 \\
床 Woman's bed
\end{tabular} \\
\hline & $\begin{array}{l}\text { Dipper for } \\
\text { liquids }\end{array}$ & \begin{tabular}{|l} 
九 \\
河 JIUHE
\end{tabular} & 晉 JIN & 貫 \\
\hline & $\begin{array}{l}\text { Dipper for } \\
\text { solids }\end{array}$ & 相 Celestial spear & 鄭 ZHENG & $\begin{array}{ll}t & \\
\text { 公 } & \text { Seven } \\
& \text { excellencies }\end{array}$ \\
\hline $\begin{array}{l}\text { 列 } \\
\text { 肆 }\end{array}$ & Jewel market & 齋 QI & 周 $\mathrm{ZHOU}$ & \\
\hline 車 & $\begin{array}{l}\text { Commodity } \\
\text { market }\end{array}$ & 䞟 WUYUE & 秦 QIN & \\
\hline $\begin{array}{l}\text { 市 } \\
\text { 樓 }\end{array}$ & $\begin{array}{l}\text { Municipal } \\
\text { office }\end{array}$ & 徐 XU & 蜀 SHU & \\
\hline $\begin{array}{l}\text { 宗 } \\
\text { 正 }\end{array}$ & $\begin{array}{l}\text { Official for } \\
\text { royal clan }\end{array}$ & $\begin{array}{l}\text { 東 } \\
\text { 海 DONGHAI }\end{array}$ & 巴 BA & \\
\hline 宗 & $\begin{array}{l}\text { Official of } \\
\text { Religious } \\
\text { Ceremonies }\end{array}$ & 燕 YAN & 梁 IANG & \\
\hline 宗 & $\begin{array}{l}\text { Patriarchal } \\
\text { clan }\end{array}$ & 南 & 楚 CHU & \\
\hline $\begin{array}{l}\text { 帛 } \\
\text { 度 }\end{array}$ & Textile ruler & 宋 SONG & 韓 HAN & \\
\hline $\begin{array}{l}\text { 屠 } \\
\text { 胙聿 }\end{array}$ & $\begin{array}{l}\text { Butcher's } \\
\text { shops }\end{array}$ & & & \\
\hline
\end{tabular}


Table 4. Seven Mansions which belong to Azure Dragon

\begin{tabular}{|c|c|c|c|c|c|c|}
\hline $\begin{array}{c}\text { 角宿 } \\
\text { Horn Mansion }\end{array}$ & $\begin{array}{c}\text { 宇宿 } \\
\text { Neck } \\
\text { Mansion }\end{array}$ & $\begin{array}{c}\text { 氐宿 } \\
\text { Root } \\
\text { Mansion }\end{array}$ & $\begin{array}{c}\text { 房宿 } \\
\text { Room } \\
\text { Mansion }\end{array}$ & $\begin{array}{c}\text { 心宿 } \\
\text { Heart } \\
\text { Mansion }\end{array}$ & $\begin{array}{c}\text { 尾宿 } \\
\text { Tail } \\
\text { Mansion }\end{array}$ & $\begin{array}{c}\text { 箕宿 } \\
\text { Winnowing- } \\
\text { basket } \\
\text { Mansion }\end{array}$ \\
\hline $\begin{array}{c}\text { 角 } \\
\text { Horn }\end{array}$ & $\begin{array}{c}\text { 穴 } \\
\text { Neck }\end{array}$ & $\begin{array}{c}\text { 氏 } \\
\text { Root }\end{array}$ & $\begin{array}{c}\text { 房 } \\
\text { Room }\end{array}$ & $\begin{array}{c}\text { 心 } \\
\text { Heart }\end{array}$ & $\begin{array}{c}\text { 尾 } \\
\text { Tail }\end{array}$ & $\begin{array}{c}\text { 箕 } \\
\text { Winnowing } \\
\text { basket }\end{array}$ \\
\hline $\begin{array}{c}\text { 平道 } \\
\text { Flat road }\end{array}$ & $\begin{array}{c}\text { 大角 } \\
\text { Great horn }\end{array}$ & $\begin{array}{l}\text { 圥池 } \\
\text { Boats and } \\
\text { lake }\end{array}$ & $\begin{array}{l}\text { 鉤鈴 } \\
\text { Lock }\end{array}$ & $\mid$\begin{tabular}{c|} 
積卒 \\
Group of \\
soldiers
\end{tabular} & $\begin{array}{c}\text { 神宮 } \\
\text { Changing } \\
\text { room }\end{array}$ & $\begin{array}{c}\text { 中山 } \\
\text { Zhongshan }\end{array}$ \\
\hline $\begin{array}{c}\text { 天田 } \\
\text { Celestial } \\
\text { farmland }\end{array}$ & $\begin{array}{c}\text { 左執法 } \\
\text { Left law } \\
\text { administrator }\end{array}$ & $\begin{array}{c}\text { 帝席 } \\
\text { Mattress of } \\
\text { the emperor }\end{array}$ & $\begin{array}{c}\text { 鍵閉 } \\
\text { Door bolt }\end{array}$ & & $\begin{array}{l}\text { 天江 } \\
\text { Celestial } \\
\text { river }\end{array}$ & $\begin{array}{c}\text { 杵 } \\
\text { Pestle }\end{array}$ \\
\hline $\begin{array}{c}\text { 周鼎 } \\
\text { Tripod of the } \\
\text { ZHOU }\end{array}$ & $\begin{array}{c}\text { 右攝提 } \\
\text { Left } \\
\text { conductor }\end{array}$ & $\begin{array}{c}\text { 梗河 } \\
\text { Celestial } \\
\text { lance }\end{array}$ & $\begin{array}{c}\text { 罪 } \\
\text { Punishment }\end{array}$ & & $\begin{array}{c}\text { 愽説 } \\
\text { FUYUE }\end{array}$ & \\
\hline $\begin{array}{c}\text { 進賢 } \\
\text { Recommending } \\
\text { virtuous man }\end{array}$ & $\begin{array}{c}\text { 折威 } \\
\text { Executions }\end{array}$ & $\begin{array}{c}\text { 招搖 } \\
\text { Twinkling } \\
\text { indicator }\end{array}$ & $\begin{array}{c}\text { 束咸 } \\
\text { Eastern door }\end{array}$ & & $\begin{array}{l}\text { 魚 } \\
\text { Fish }\end{array}$ & \\
\hline $\begin{array}{c}\text { 天門 } \\
\text { Celestial gate }\end{array}$ & $\begin{array}{l}\text { 頓頑 } \\
\text { Trials }\end{array}$ & $\begin{array}{c}\text { 天乳 } \\
\text { Celestial milk }\end{array}$ & $\begin{array}{c}\text { 西咸 } \\
\text { Western door }\end{array}$ & & $\begin{array}{c}\text { 龜 } \\
\text { Tortoise }\end{array}$ & \\
\hline $\begin{array}{c}\text { 平 } \\
\text { Judging }\end{array}$ & $\begin{array}{c}\text { 陽門 } \\
\text { Gate of } \\
\text { YANG }\end{array}$ & $\begin{array}{c}\text { 天輻 } \\
\text { Celestial } \\
\text { spokes }\end{array}$ & $\begin{array}{c}\text { 日 } \\
\text { Sun }\end{array}$ & & & \\
\hline $\begin{array}{c}\text { 庫椤 } \\
\text { Arsenal }\end{array}$ & & $\begin{array}{c}\text { 陣車 } \\
\text { Battle } \\
\text { chariots }\end{array}$ & $\begin{array}{c}\text { 從官 } \\
\text { Retinue }\end{array}$ & & & \\
\hline $\begin{array}{c}\text { 柱 } \\
\text { Pillars }\end{array}$ & & $\begin{array}{c}\text { 車騎 } \\
\text { Chariots and } \\
\text { cavalry }\end{array}$ & & & & \\
\hline $\begin{array}{c}\text { 衡 } \\
\text { Railings }\end{array}$ & & $\begin{array}{c}\text { 騎陣將軍 } \\
\text { Chariot and } \\
\text { Cavalry } \\
\text { general }\end{array}$ & & & & \\
\hline $\begin{array}{c}\text { 南門 } \\
\text { Southern gate }\end{array}$ & & $\begin{array}{c}\text { 騎官 } \\
\text { Imperial } \\
\text { guards }\end{array}$ & & & & \\
\hline
\end{tabular}


Table 5. The Seven Mansions which belong to Vermilion Bird

\begin{tabular}{|c|c|c|c|c|c|c|c|}
\hline 井宿 Well & Mansion & $\begin{array}{c}\text { 鬼宿 } \\
\text { Ghosts } \\
\text { Mansion }\end{array}$ & $\begin{array}{c}\text { 柳宿 } \\
\text { Willow } \\
\text { Mansion }\end{array}$ & $\begin{array}{c}\text { 星宿 } \\
\text { Star } \\
\text { Mansion }\end{array}$ & $\begin{array}{c}\text { 張宿 } \\
\text { Extended } \\
\text { Net } \\
\text { Mansion }\end{array}$ & $\begin{array}{c}\text { 翼宿 } \\
\text { Wings } \\
\text { Mansion }\end{array}$ & $\begin{array}{c}\text { 軫宿 } \\
\text { Chariot } \\
\text { Mansion }\end{array}$ \\
\hline $\begin{array}{c}\text { 井 } \\
\text { Well }\end{array}$ & $\begin{array}{c}\text { 四瀆 } \\
\text { Four } \\
\text { channels }\end{array}$ & $\begin{array}{c}\text { 鬼 } \\
\text { Ghosts }\end{array}$ & $\begin{array}{c}\text { 柳 } \\
\text { Willow }\end{array}$ & $\begin{array}{c}\text { 星 } \\
\text { Star }\end{array}$ & $\begin{array}{c}\text { 張 } \\
\text { Extended } \\
\text { net }\end{array}$ & $\begin{array}{c}\text { 翼 } \\
\text { Wings }\end{array}$ & $\begin{array}{c}\text { 軫 } \\
\text { Chariot }\end{array}$ \\
\hline $\begin{array}{c}\text { 銭 } \\
\text { Battle axe }\end{array}$ & $\begin{array}{c}\text { 閶丘 } \\
\text { Palace gate }\end{array}$ & $\begin{array}{c}\text { 積尸 } \\
\text { Cumulative } \\
\text { corpses }\end{array}$ & $\begin{array}{c}\text { 酒旗 } \\
\text { Banner } \\
\text { of wine } \\
\text { shop }\end{array}$ & $\begin{array}{c}\text { 天相 } \\
\text { Celestial } \\
\text { premier }\end{array}$ & $\begin{array}{c}\text { 天廟 } \\
\text { Celestial } \\
\text { Temple }\end{array}$ & $\begin{array}{c}\text { 束旊 } \\
\text { DONGOU }\end{array}$ & $\begin{array}{c}\text { 左轄 } \\
\text { Left } \\
\text { linchpin }\end{array}$ \\
\hline $\begin{array}{l}\text { 水府 } \\
\text { Official for } \\
\text { irrigation }\end{array}$ & $\begin{array}{c}\text { 軍市 } \\
\text { Market for } \\
\text { the soldiers }\end{array}$ & $\begin{array}{c}\text { 篗 } \\
\text { Beacon fire }\end{array}$ & & $\begin{array}{c}\text { 天䅼 } \\
\text { Celestial } \\
\text { cereals }\end{array}$ & & & $\begin{array}{c}\text { 右轄 } \\
\text { Right } \\
\text { linchpin }\end{array}$ \\
\hline $\begin{array}{c}\text { 天樽 } \\
\text { Celestial } \\
\text { wine cup }\end{array}$ & $\begin{array}{c}\text { 野雜 } \\
\text { Wild } \\
\text { cockerel }\end{array}$ & $\begin{array}{c}\text { 外廚 } \\
\text { Outer } \\
\text { kitchen }\end{array}$ & & $\begin{array}{c}\text { 軒轅 } \\
\text { Xuanyuan }\end{array}$ & & & $\begin{array}{c}\text { 長沙 } \\
\text { Changsha }\end{array}$ \\
\hline $\begin{array}{l}\text { 五諸侯 } \\
\text { Five feudal } \\
\text { kings }\end{array}$ & $\begin{array}{c}\text { 天狼 } \\
\text { Celestial } \\
\text { wolf }\end{array}$ & $\begin{array}{c}\text { 天記 } \\
\text { Judge to } \\
\text { estimate } \\
\text { the age of } \\
\text { animals }\end{array}$ & & $\begin{array}{c}\text { 御女 } \\
\text { Maids-in- } \\
\text { waiting }\end{array}$ & & & $\begin{array}{c}\text { 青丘 } \\
\text { Green } \\
\text { hill }\end{array}$ \\
\hline $\begin{array}{c}\text { 北河 } \\
\text { North river }\end{array}$ & $\begin{array}{c}\text { 丈人 } \\
\text { Grandfather }\end{array}$ & $\begin{array}{c}\text { 月 } \\
\text { Moon }\end{array}$ & & $\begin{array}{l}\text { 内平 } \\
\text { High } \\
\text { judge }\end{array}$ & & & $\begin{array}{c}\text { 軍門 } \\
\text { Military } \\
\text { Gate }\end{array}$ \\
\hline $\begin{array}{c}\text { 積水 } \\
\text { Accumulated } \\
\text { water }\end{array}$ & $\begin{array}{l}\text { 子 } \\
\text { Son }\end{array}$ & $\begin{array}{c}\text { 天狗 } \\
\text { Celestial } \\
\text { dog }\end{array}$ & & & & & \begin{tabular}{|c|} 
土司空 \\
Master \\
of Con- \\
struction
\end{tabular} \\
\hline $\begin{array}{c}\text { 積薪 } \\
\text { Pile of } \\
\text { firewood }\end{array}$ & $\begin{array}{c}\text { 孫 } \\
\text { Grandson }\end{array}$ & $\begin{array}{c}\text { 天社 } \\
\text { Celestial } \\
\text { earth god's } \\
\text { temple }\end{array}$ & & & & & \begin{tabular}{|c|} 
器府 \\
House for \\
Musical \\
Instru- \\
ments
\end{tabular} \\
\hline $\begin{array}{c}\text { 水位 } \\
\text { Water level }\end{array}$ & $\begin{array}{c}\text { 老人 } \\
\text { Old man }\end{array}$ & & & & & & \\
\hline $\begin{array}{c}\text { 南河 } \\
\text { South river }\end{array}$ & $\begin{array}{c}\text { 弧矢 } \\
\text { Bow and } \\
\text { arrow }\end{array}$ & & & & & & \\
\hline
\end{tabular}


Table 6. The Seven Mansions of the White Tiger

\begin{tabular}{|c|c|c|c|c|c|c|c|}
\hline $\begin{array}{c}\text { 奎宿 } \\
\text { Legs } \\
\text { Mansion }\end{array}$ & $\begin{array}{c}\text { 婁宿 } \\
\text { Bond } \\
\text { Mansion }\end{array}$ & $\begin{array}{c}\text { 胃宿 } \\
\text { Stomach } \\
\text { Mansion }\end{array}$ & $\begin{array}{c}\text { 帠宿 } \\
\text { Hairy } \\
\text { Head } \\
\text { Mansion }\end{array}$ & 畢宿 N & et Mansion & $\begin{array}{c}\text { 觜宿 } \\
\text { Turtle } \\
\text { Beak } \\
\text { Mansion }\end{array}$ & \begin{tabular}{|c} 
參宿 \\
Three Stars \\
Mansion
\end{tabular} \\
\hline $\begin{array}{c}\text { 奎 } \\
\text { Legs }\end{array}$ & $\begin{array}{c}\text { 婁 } \\
\text { Bond }\end{array}$ & $\begin{array}{c}\text { 胃 } \\
\text { Stomach }\end{array}$ & $\begin{array}{c}\stackrel{9}{\eta^{\prime}} \\
\text { Hairy head }\end{array}$ & $\begin{array}{c}\text { 畢 } \\
\text { Net }\end{array}$ & $\begin{array}{c}\text { 天關 } \\
\text { Celestial } \\
\text { gate }\end{array}$ & $\begin{array}{c}\text { 觜 } \\
\text { Turtle } \\
\text { beak }\end{array}$ & $\begin{array}{c}\text { 參 } \\
\text { Three stars }\end{array}$ \\
\hline $\begin{array}{c}\text { 王良 } \\
\text { Wangliang }\end{array}$ & $\begin{array}{c}\text { 天大將軍 } \\
\text { Great } \\
\text { general of } \\
\text { the } \\
\text { heaven }\end{array}$ & $\begin{array}{c}\text { 大陵 } \\
\text { Mausoleum }\end{array}$ & $\begin{array}{c}\text { 天阿 } \\
\text { Celestial } \\
\text { river }\end{array}$ & $\begin{array}{c}\text { 附耳 } \\
\text { Whisper }\end{array}$ & $\begin{array}{c}\text { 天節 } \\
\text { Celestial } \\
\text { tally }\end{array}$ & $\begin{array}{c}\text { 司怪 } \\
\text { Deity in } \\
\text { charge of } \\
\text { monsters }\end{array}$ & $\begin{array}{c}\text { 伐 } \\
\text { Punishment }\end{array}$ \\
\hline $\begin{array}{c}\text { 策 } \\
\text { Whip }\end{array}$ & $\begin{array}{c}\text { 左更 } \\
\text { Official in } \\
\text { charge of } \\
\text { forest }\end{array}$ & $\begin{array}{c}\text { 積尸 } \\
\text { Heaps of } \\
\text { corpses }\end{array}$ & $\begin{array}{l}\text { 齊 } \\
\mathrm{QI}\end{array}$ & $\begin{array}{c}\text { 天街 } \\
\text { Celestial } \\
\text { street }\end{array}$ & $\begin{array}{c}\text { 九州殊口 } \\
\text { Interpreters } \\
\text { of nine } \\
\text { dialects }\end{array}$ & \begin{tabular}{|c} 
座旗 \\
Seat flags
\end{tabular} & $\begin{array}{c}\text { 玉井 } \\
\text { Jade well }\end{array}$ \\
\hline $\begin{array}{c}\text { 附路 } \\
\text { Auxiliary } \\
\text { road }\end{array}$ & $\begin{array}{c}\text { 右更 } \\
\text { Official in } \\
\text { charge of } \\
\text { pasturing }\end{array}$ & $\begin{array}{c}\text { 天船 } \\
\text { Celestial } \\
\text { boat }\end{array}$ & $\begin{array}{l}\text { 卷舌 } \\
\text { Rolled } \\
\text { tongue }\end{array}$ & $\begin{array}{c}\text { 天高 } \\
\text { Celestial } \\
\text { high } \\
\text { terrace }\end{array}$ & $\begin{array}{c}\text { 參旗 } \\
\text { Banner of } \\
\text { three stars }\end{array}$ & & $\begin{array}{l}\text { 鉄鈛 } \\
\text { Axe }\end{array}$ \\
\hline $\begin{array}{c}\text { 軍南門 } \\
\text { Southern } \\
\text { military } \\
\text { gate }\end{array}$ & $\begin{array}{c}\text { 天含 } \\
\text { Square } \\
\text { celestial } \\
\text { granary }\end{array}$ & $\begin{array}{c}\text { 積水 } \\
\text { Stored } \\
\text { water }\end{array}$ & $\begin{array}{c}\text { 天䜛 } \\
\text { Celestial } \\
\text { slander }\end{array}$ & $\begin{array}{c}\text { 諸王 } \\
\text { Feudal } \\
\text { kings }\end{array}$ & $\begin{array}{c}\text { 九斿 } \\
\text { Imperial } \\
\text { military } \\
\text { flag }\end{array}$ & & $\begin{array}{c}\text { 軍井 } \\
\text { Military } \\
\text { well }\end{array}$ \\
\hline $\begin{array}{c}\text { 閣道 } \\
\text { Flying } \\
\text { corridor }\end{array}$ & $\begin{array}{c}\text { 天庚 } \\
\text { Ricks of } \\
\text { grain }\end{array}$ & $\begin{array}{c}\text { 天店 } \\
\text { Celestial } \\
\text { foodstuffs }\end{array}$ & $\begin{array}{c}\text { 礪石 } \\
\text { Whetstone }\end{array}$ & $\begin{array}{c}\text { 五車 } \\
\text { Five } \\
\text { chariots }\end{array}$ & $\begin{array}{c}\text { 天園 } \\
\text { Celestial } \\
\text { orchard }\end{array}$ & & $\begin{array}{c}\text { 屏 } \\
\text { Screen }\end{array}$ \\
\hline $\begin{array}{l}\text { 外屏 } \\
\text { Outer } \\
\text { fence }\end{array}$ & & $\begin{array}{c}\text { 天囷 } \\
\text { Circular } \\
\text { celestial } \\
\text { granary }\end{array}$ & $\begin{array}{c}\text { 天陰 } \\
\text { Celestial } \\
\text { YIN force }\end{array}$ & $\begin{array}{c}\text { 柱 } \\
\text { Pillars }\end{array}$ & & & $\begin{array}{c}\text { 廁 } \\
\text { Toilet }\end{array}$ \\
\hline $\begin{array}{c}\text { 天泪 } \\
\text { Celestial } \\
\text { pigsty }\end{array}$ & & & $\begin{array}{l}\text { 留蒿 } \\
\text { Hay }\end{array}$ & $\begin{array}{c}\text { 威池 } \\
\text { Pool of } \\
\text { harmony }\end{array}$ & & & $\begin{array}{c}\text { 屎 } \\
\text { Excrement }\end{array}$ \\
\hline $\begin{array}{c}\text { 土司空 } \\
\text { Master of } \\
\text { construc- } \\
\text { tions }\end{array}$ & & & $\begin{array}{c}\text { 天苑 } \\
\text { Celestial } \\
\text { meadows }\end{array}$ & $\begin{array}{c}\text { 天潢 } \\
\text { Celestial } \\
\text { pier }\end{array}$ & & & \\
\hline
\end{tabular}


Table 7. The Seven Mansions of the Murky Warrior

\begin{tabular}{|c|c|c|c|c|c|c|c|}
\hline $\begin{array}{c}\text { 斗宿 } \\
\text { Dipper } \\
\text { Mansion }\end{array}$ & $\begin{array}{c}\text { 牛宿 } \\
\text { Ox } \\
\text { Mansion }\end{array}$ & 女宿 Girl & Mansion & $\begin{array}{c}\text { 虛宿 } \\
\text { Emptiness } \\
\text { Mansion }\end{array}$ & $\begin{array}{c}\text { 危宿 } \\
\text { Rooftop } \\
\text { Mansion }\end{array}$ & $\begin{array}{c}\text { 室宿 } \\
\text { Encampment } \\
\text { Mansion }\end{array}$ & $\begin{array}{c}\text { 壁宿 } \\
\text { Wall } \\
\text { Mansion }\end{array}$ \\
\hline $\begin{array}{c}\text { 斗 } \\
\text { Dipper }\end{array}$ & $\begin{array}{l}\text { 牛 } \\
\mathrm{Ox}\end{array}$ & $\begin{array}{c}\text { 女 } \\
\text { Girl }\end{array}$ & $\begin{array}{c}\text { 趙 } \\
\mathrm{ZHAO}\end{array}$ & $\begin{array}{c}\text { 虛宿 } \\
\text { Emptiness }\end{array}$ & $\begin{array}{c}\text { 危 } \\
\text { Rooftop }\end{array}$ & $\begin{array}{c}\text { 室 } \\
\text { Encampment }\end{array}$ & 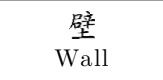 \\
\hline $\begin{array}{c}\text { 天圇 } \\
\text { Celestial } \\
\text { keyhole }\end{array}$ & $\begin{array}{c}\text { 天桴 } \\
\text { Celestial } \\
\text { drumstick }\end{array}$ & $\begin{array}{c}\text { 離珠 } \\
\text { Pearls on } \\
\text { ladies' } \\
\text { wear }\end{array}$ & $\begin{array}{c}\text { 越 } \\
Y U E\end{array}$ & $\begin{array}{c}\text { 司命 } \\
\text { Deified } \\
\text { judge of } \\
\text { life }\end{array}$ & $\begin{array}{l}\text { 境墓 } \\
\text { Tomb }\end{array}$ & $\begin{array}{c}\text { 離宫 } \\
\text { Resting } \\
\text { palace }\end{array}$ & $\begin{array}{c}\text { 天㩔 } \\
\text { Celestial } \\
\text { stable }\end{array}$ \\
\hline $\begin{array}{c}\text { 天弁 } \\
\text { Market officer }\end{array}$ & $\begin{array}{c}\text { 河鼓 } \\
\text { Drum at } \\
\text { the river }\end{array}$ & $\begin{array}{l}\text { 敗瓜 } \\
\text { Rotten } \\
\text { gourd }\end{array}$ & $\begin{array}{c}\text { 左攝提 } \\
\text { Right } \\
\text { conductor }\end{array}$ & $\begin{array}{c}\text { 司祿 } \\
\text { Deified } \\
\text { judge of } \\
\text { rank }\end{array}$ & $\begin{array}{c}\text { 蓋屋 } \\
\text { Roofing }\end{array}$ & $\begin{array}{c}\text { 螣蛇 } \\
\text { Flying } \\
\text { serpent }\end{array}$ & $\begin{array}{l}\text { 土公 } \\
\text { Official for } \\
\text { earthworks } \\
\text { and } \\
\text { buildings }\end{array}$ \\
\hline $\begin{array}{c}\text { 建 } \\
\text { Establishment }\end{array}$ & $\begin{array}{c}\text { 左旗 } \\
\text { Left flag }\end{array}$ & $\begin{array}{l}\text { 瓠瓜 } \\
\text { Good } \\
\text { gourd }\end{array}$ & $\begin{array}{c}\text { 鄭 } \\
\text { ZHENG }\end{array}$ & $\begin{array}{c}\text { 司危 } \\
\text { Deified } \\
\text { judge of } \\
\text { disaster } \\
\text { and good } \\
\text { fortune }\end{array}$ & $\begin{array}{c}\text { 虚梁 } \\
\text { Temple }\end{array}$ & $\begin{array}{c}\text { 雷電 } \\
\text { Thunder and } \\
\text { lightning }\end{array}$ & $\begin{array}{c}\text { 霹霍 } \\
\text { Thunderbolt }\end{array}$ \\
\hline $\begin{array}{c}\text { 天雞 } \\
\text { Celestial cock }\end{array}$ & $\begin{array}{c}\text { 右旗 } \\
\text { Right flag }\end{array}$ & $\begin{array}{c}\text { 天津 } \\
\text { Celestial } \\
\text { ford }\end{array}$ & $\begin{array}{c}\text { 魏 } \\
\text { WEI }\end{array}$ & $\begin{array}{c}\text { 司非 } \\
\text { Deified } \\
\text { judge of } \\
\text { right and } \\
\text { wrong }\end{array}$ & $\begin{array}{c}\text { 天銭 } \\
\text { Celestial } \\
\text { money }\end{array}$ & $\begin{array}{l}\text { 土公吏 } \\
\text { Official for } \\
\text { materials } \\
\text { supply }\end{array}$ & $\begin{array}{l}\text { 雲雨 } \\
\text { Cloud and } \\
\text { rain }\end{array}$ \\
\hline $\begin{array}{c}\text { 狗 } \\
\text { Dog }\end{array}$ & $\begin{array}{c}\text { 織女 } \\
\text { Weaving } \\
\text { girl }\end{array}$ & $\begin{array}{c}\text { 奚仲 } \\
\text { XIZHONG }\end{array}$ & $\begin{array}{c}\text { 韓 } \\
\mathrm{HAN}\end{array}$ & $\begin{array}{c}\text { 哭 } \\
\text { Crying }\end{array}$ & 人 & $\begin{array}{c}\text { 壆壁陣 } \\
\text { The line of } \\
\text { ramparts }\end{array}$ & $\begin{array}{l}\text { 鉄镍 } \\
\text { Sickle }\end{array}$ \\
\hline $\begin{array}{c}\text { 狗國 } \\
\text { Territory of } \\
\text { dogs }\end{array}$ & $\begin{array}{c}\text { 漸薹 } \\
\text { Clepsydra } \\
\text { terrace }\end{array}$ & $\begin{array}{c}\text { 扶筐 } \\
\text { Basket for } \\
\text { mulberry } \\
\text { leaves }\end{array}$ & $\begin{array}{c}\text { 晉 } \\
\text { JIN }\end{array}$ & $\begin{array}{c}\text { 泣 } \\
\text { Weeping }\end{array}$ & $\begin{array}{c}\text { 杵 } \\
\text { Pestle }\end{array}$ & $\begin{array}{c}\text { 羽林軍 } \\
\text { Palace guard }\end{array}$ & \\
\hline $\begin{array}{c}\text { 天滆 } \\
\text { Celestial } \\
\text { spring }\end{array}$ & $\begin{array}{c}\text { 杽道 } \\
\text { Imperial } \\
\text { passageway }\end{array}$ & $\begin{array}{c}\text { 十二國 } \\
\text { Twelve } \\
\text { countries }\end{array}$ & $\begin{array}{c}\text { 燕 } \\
\text { YAN }\end{array}$ & $\begin{array}{c}\text { 璃瑜 } \\
\text { Jade } \\
\text { ornament } \\
\text { on ladies' } \\
\text { wear }\end{array}$ & $\begin{array}{c}\text { 兒 } \\
\text { Mortar }\end{array}$ & $\begin{array}{c}\text { 天綱 } \\
\text { Materials for } \\
\text { Making } \\
\text { Tents }\end{array}$ & \\
\hline $\begin{array}{l}\text { 農丈人 } \\
\text { Peasant }\end{array}$ & $\begin{array}{c}\text { 羅堰 } \\
\text { Networks of } \\
\text { dykes }\end{array}$ & $\begin{array}{c}\text { 周 } \\
\mathrm{ZHOU}\end{array}$ & & $\begin{array}{c}\text { 天罣城 } \\
\text { Celestial } \\
\text { ramparts }\end{array}$ & $\begin{array}{c}\text { 車府 } \\
\text { Big yard } \\
\text { for } \\
\text { chariots }\end{array}$ & $\begin{array}{c}\text { 北落師門 } \\
\text { North gate of } \\
\text { the military } \\
\text { camp }\end{array}$ & \\
\hline \multirow[t]{2}{*}{$\begin{array}{c}\text { 鰵 } \\
\text { River turtle }\end{array}$} & $\begin{array}{c}\text { 天田 } \\
\text { Celestial } \\
\text { farmland }\end{array}$ & $\begin{array}{c}\text { 秦 } \\
\text { QIN }\end{array}$ & & $\begin{array}{c}\text { 敗鼠 } \\
\text { Decayed } \\
\text { Mortar }\end{array}$ & $\begin{array}{c}\text { 造父 } \\
\text { ZAOFU }\end{array}$ & $\begin{array}{c}\text { 糠 } \\
\text { Chaff }\end{array}$ & \\
\hline & $\begin{array}{c}\text { 九坎 } \\
\text { Nine water } \\
\text { wells }\end{array}$ & $\begin{array}{c}\text { 代 } \\
\text { DAI }\end{array}$ & & & $\begin{array}{c}\text { 天鉤 } \\
\text { Celestial } \\
\text { hook }\end{array}$ & $\begin{array}{c}\text { 八魁 } \\
\text { Net for } \\
\text { catching } \\
\text { birds }\end{array}$ & \\
\hline
\end{tabular}

